\title{
Whole-body MRI and MRA for evaluation of the prevalence of atherosclerosis in a cohort of subjectively healthy individuals
}

\author{
M. Laible • S. O. Schoenberg • S. Weckbach • \\ M. Lettau • E. Winnik • J. Bischof • R. Franke • \\ M. Reiser • H. Kramer
}

Received: 6 December 2011 /Revised: 23 May 2012 / Accepted: 4 June 2012 /Published online: 27 July 2012

(C) The Author(s) 2012. This article is published with open access at Springerlink.com

\begin{abstract}
Objectives To assess the prevalence of cardiovascular findings in asymptomatic individuals by means of 1.5 - $\mathrm{T}$ wholebody magnetic resonance imaging and angiography.

Methods A cohort of 138 individuals (118 men, 20 women) with a mean age of 54 years $(\mathrm{SD} \pm 7.55)$ was referred to whole-body MRI at 1.5-T, including contrast-enhanced whole-body MR angiography (MRA) and cardiac MRI. A total of 2,065/2,070 vessel segments (99.8\%) and cardiac function were evaluated.
\end{abstract}

M. Laible $\cdot$ S. Weckbach $\cdot$ M. Reiser $\cdot$ H. Kramer

Department of Clinical Radiology, University Hospital Munich, Grosshadern Campus,

Munich, Germany

\section{Laible $(\square)$}

Department of Neurology, University Hospital Heidelberg,

Im Neuenheimer Feld 400,

69120 Heidelberg, Germany

e-mail: monalaible@googlemail.com

\section{S. O. Schoenberg}

Department of Clinical Radiology and Nuclear Medicine, University Hospital Mannheim, Medical Faculty Mannheim, University of Heidelberg,

Mannheim, Germany

\section{Lettau}

Department of Neuroradiology, University Hospital Freiburg, Freiburg, Germany

E. Winnik $\cdot$ R. Franke

Siemens Healthcare, Siemens AG,

Erlangen, Germany

J. Bischof

Gesundheitsdienst BMW AG, BMW AG,

Munich, Germany
Results Approximately one-fourth of the participating individuals had vascular abnormalities. In 17 subjects $(12.3 \%$ of all subjects) significant luminal narrowing was observed in at least one vascular segment. Luminal narrowing (mild to severe) was observed in $1(0.7 \%$ of all subjects respectively) of the renal arteries, 7 (5.0\%) of the carotid arteries, and 3 $(2.2 \%)$ of the pelvic and upper leg arteries, and in 17 segments $(12.3 \%)$ of arteries in the lower leg.

In cardiac function and perfusion imaging, wall motion disorders were observed in six patients $(4.3 \%)$, with additional delayed enhancement and isolated delayed enhancement present in two cases. Functional parameters differed from reference values in 55 cases.

Conclusions Even in an asymptomatic cohort of middleaged predominantly male individuals, atherosclerotic disease is not uncommon and is detectable by whole-body MRI.

Main Messages

- In middle-aged predominantly male individuals, atherosclerotic disease is not uncommon.

- Even in an asymptomatic collective, approximately one fourth had vascular abnormalities.

- Using whole-body MR angiography (MRA), 99.8\% of 2,070 vessel segments could be evaluated.

Keywords Cardiovascular disease · Epidemiology · Magnetic resonance angiography

\section{Introduction}

The first manifestation of cardiovascular disease may occur as a fatal event such as myocardial infarction or stroke [27]. Thus, early detection and diagnosis of cardiovascular 
disease allow the initiation of secondary preventive measures, which seems to be most favorable in early disease stages. This has been shown to be beneficial for risk reduction related to subsequent events such as myocardial infarction and sudden cardiac death $[1,2]$. The occurrence of atherosclerotic vascular disease in one vascular territory makes a manifestation in further vascular regions highly probable [3]. While complete atherosclerotic lesion reversal is usually not possible, disease progression can be controlled and decelerated.

Various diagnostic methods have been used to evaluate the presence of atherosclerosis in humans; however, only certain modalities are adequate for use in asymptomatic persons. Magnetic resonance angiography (MRA) is a compelling modality that lacks ionizing radiation, is non-invasive, and has less risk of nephrotoxicity compared with iodinated contrast agents in patients with sufficient renal function (GFR > $30 \mathrm{ml} / \mathrm{min}$ ). In addition, the feasibility of whole-body MRI examinations has been documented recently by several studies [4, 5, 11-16]. For tumor imaging, whole-body MRI (wb-MRI) has also been introduced and has comparable accuray to PET$\mathrm{CT}$ for tumor and metastasis detection. However, wb-MRI has been predominantly used for cancer staging or follow-up examinations in subjects with a history of malignant disease and not as a primary screening tool.

Whole-body MR angiography is also feasible, and prior studies have shown that whole-body contrast-enhanced MRangiography (wb-CE-MRA) has a high sensitivity and specificity for estimating the extent of atherosclerosis in patients with vascular disease compared to digital subtraction angiography (DSA) as the gold standard $[4,5]$.Therefore, the primary intent of this study was not to determine the diagnostic accuracy of wb-MRA, but to describe whole-body MRI findings in terms of atherosclerotic changes in a cohort of asymptomatic middle-aged individuals in Germany in an observational prospective study.

\section{Materials and methods}

\section{Study subjects}

Our study protocol was presented to and approved by the local Institutional Review Board. Subjectively asymptomatic subjects that participated in a routine health screening program and for this purpose were regularly seen by their medical practitioners were eligible for inclusion. All healthy attendants of the routine health screening program of two major German companies were asked to participate in wbMRI/-MRA on a voluntary basis. For a precise pre-selection on the basis of self-reported patient history, we excluded all subjects with a preliminary history or symptoms of cardiovascular disease.
One hundred thirty-eight individuals, 118 males and 20 females with a mean age of 54 years (age range, 39-74 years), consented to participate, met the inclusion criteria and were thus included between April 2003 and January 2005. For the age distribution of the participants, see Tables 1 and 2. Each subject gave oral and written informed consent. The authors were not financially supported by the referring health practitioner's companies.

As subjectively reported spontaneously by themselves, the participants' health practitioners in parallel had not documented any cardiovascular or malignant disease, but reported risk factors such as familiar predisposition, cigarette smoking, lack of exercise, etc. The basic health examination consisted of electrocardiography at rest, stress testing using an ergometer, transthoracic echocardiography, duplex ultrasound of the carotid arteries, and chest radiography. Further, an abdominal ultrasound was performed, as was blood analysis including a complete blood cell count and differential blood cell count. The findings of the wholebody MRI were classified as follows. Relevant findings were defined as those that led to a referral to a physician, and further diagnostic workup or therapy (e.g., signs of myocarditis). Non-relevant findings were defined as incidental pathological findings that did not require further monitoring (e.g., a solitary renal cyst).

Whenever data on routine or dedicated control examinations were available, MRI findings were compared on a case-by-case basis.

\section{MRI systems}

While conducting the study, two different MRI systems were used. The first 36 consecutive subjects (group 1) were imaged using a standard clinical 1.5-T MRI system equipped with eight receiver channels (Magnetom Sonata Maestro Class, Siemens Medical Solutions, Erlangen, Germany). The following 102 consecutive subjects (group 2) were evaluated using a 1.5-T whole-body MRI system equipped with 32 receiver channels (Magnetom Avanto, Siemens Medical Solutions, Erlangen, Germany).

Since both systems were equipped with multiple receiver channels and multi-element surface coils, use of parallel imaging techniques (integrated parallel acquisition techniques,

Table 1 Data of participants $(n=138)$

\begin{tabular}{lllll}
\hline & $\mathrm{N}$ & $\begin{array}{l}\text { Median age } \\
\text { (years) }\end{array}$ & $\begin{array}{l}\text { Average } \\
\text { (years) }\end{array}$ & $\begin{array}{l}\text { Standard } \\
\text { deviation (years) }\end{array}$ \\
\hline Men & 118 & 56.0 & 55.6 & 7.3 \\
Women & 20 & 51.5 & 54.2 & 9.0 \\
Total & 138 & 56.0 & 55.4 & 7.6 \\
\hline
\end{tabular}


Table 2 Graduation according to age

\begin{tabular}{lllll}
\hline & $\begin{array}{l}\text { Up to } 49 \\
\text { years }\end{array}$ & $\begin{array}{l}50 \text { to } 59 \\
\text { years }\end{array}$ & $\begin{array}{l}60 \text { to } 69 \\
\text { years }\end{array}$ & $\begin{array}{l}70 \text { or more } \\
\text { years }\end{array}$ \\
\hline $\begin{array}{l}\text { Number of } \\
\text { participants }\end{array}$ & 33 & 57 & 44 & 4 \\
\hline
\end{tabular}

iPAT, Siemens Medical Solutions, Erlangen, Germany) was possible with both scanner types.

\section{Whole-body MRI protocol}

To perform pre- and postcontrast imaging of the whole body, a dedicated imaging protocol had to be designed. In group 1, using a standard MR system, for imaging of the thorax, carotid arteries, and the brain, the subject was positioned head first in the magnet and the region of interest placed in the isocenter by table movement. For the second part of the MR examination, the participant was repositioned feet first in the scanner. This allowed for an examination beginning with the lower thorax down to the feet. In group 2, using a dedicated whole-body MR system, repositioning was not necessary because of the wide table movement range of up to $205 \mathrm{~cm}$. All coils necessary for the exam were positioned prior to starting the examination and separately selected during the exam when needed. After acquisition of all necessary localizers, all subjects underwent noncontrast-enhanced MR imaging of the lungs first using halfFourier RARE techniques. Second, imaging of cardiac function and perfusion was performed followed by highresolution contrast-enhanced MR angiography as specified in Table 3 for the standard MR imager and in Table 4 for the whole-body imaging system.

Table 3 MRI protocol for the 1.5-T standard MR system

\begin{tabular}{|c|c|c|}
\hline $\begin{array}{l}\text { Examination } \\
\text { time in min }\end{array}$ & Anatomic region & Sequence \\
\hline \multirow[t]{2}{*}{ Start } & Thorax and abdomen & Half-Fourier RARE \\
\hline & Heart & $\begin{array}{l}\text { Function and perfusion } \\
\text { imaging }\end{array}$ \\
\hline \multirow[t]{4}{*}{30} & Carotid arteries & MR angiography \\
\hline & Brain & $\begin{array}{l}\text { T1- and T2-weighted } \\
\text { imaging }\end{array}$ \\
\hline & Thorax & $\begin{array}{l}\text { Volumetric interpolated } \\
\text { breathhold } \\
\text { examination }\end{array}$ \\
\hline & Heart & $\begin{array}{l}\text { Delayed contrast- } \\
\text { enhanced imaging }\end{array}$ \\
\hline 60 & $\begin{array}{l}\text { Abdominal aorta, thigh, } \\
\text { and calf vessels }\end{array}$ & MR angiography \\
\hline 90 & Abdomen & $\begin{array}{l}\text { Half-Fourier RARE, } \\
\text { FLASH }\end{array}$ \\
\hline
\end{tabular}

Table 4 MRI protocol for the whole body imager

\begin{tabular}{lll}
\hline $\begin{array}{l}\text { Examination } \\
\text { time in min }\end{array}$ & Anatomic region & Sequence \\
\hline Start & Thorax and abdomen & $\begin{array}{l}\text { Half-Fourier RARE } \\
\text { Function and perfusion } \\
\text { imaging }\end{array}$ \\
& Heart & MR angiography \\
30 & Carotid arteries, calf & vessels \\
& Brain & T1-, T2-, and diffusion- \\
& weighted imaging \\
& Heart & $\begin{array}{c}\text { Delayed contrast- } \\
\text { enhanced imaging }\end{array}$ \\
& Thorax & Volumetric interpolated \\
& & breathhold \\
& & examination \\
& Abdominal aorta, thigh & MR angiography \\
& vessels & FLASH \\
90 & Abdomen & \\
\hline
\end{tabular}

Cardiac function imaging

For assessment of cardiac function, we used a real-time cardiac imaging sequence (true fast imaging with steadystate precession, TrueFISP). In this sequence, all k-space lines are acquired within two heartbeats for imaging of a single slice of the heart. Using parallel imaging techniques, the temporal resolution could be improved to $48 \mathrm{~ms}$. Miller et al. assumed a higher influence of temporal resolution to functional parameters of the left ventricle (LV) compared to the influence of spatial resolution of less than $2 \mathrm{~mm} \mathrm{[6].}$ Cardiodynamic parameters including end-diastolic volume (EDV), end-systolic volume (ESV), EF, and myocardial mass (MM) were calculated using the modified Simpson's rule (Argus Software, Siemens, Germany). All values were normalised to body surface measurements in square meters.

Myocardial perfusion imaging

First-pass perfusion imaging of the heart at rest was performed using a saturation-recovery trueFISP sequence with a parallel imaging factor of 2 . This allowed for an acquisition of four slices in a single breathhold. For myocardial perfusion imaging, a Gd-based contrast agent was used at a dose of $0.05 \mathrm{mmol}$ per kilogram of body weight. Perfusion imaging at rest can only detect severe coronary artery stenosis. However, using pharmaceutically induced stress in subjects with risk factors for cardiovascular disease but without clinical symptoms was not ethically feasible.

Cardiac delayed contrast-enhanced imaging

It is well known that after administration of Gd contrast agents, infarcted myocardium exhibits delayed hyperenhancement and 
can be imaged using an inversion recovery sequence. The performance of such a method when using magnitudereconstructed images is highly sensitive to the inversion recovery time selected. The contrast agent washes in and out of the normal perfused myocardium and infarcted tissues at different rates, giving rise to the difference in tissue T1 values and leading to the observed delayed hyperenhancement [7]. We used a phase-sensitive inversion recovery sequence as this technique enables cardiac delayed contrast-enhanced (DCE) imaging without prior scout imaging. This sequence allows for the imaging of nine slices during one breath-hold and has been shown to deliver accurate identification of the area and volume of infarction with high spatial resolution [8, 24]. For best depiction of delayed enhancement, we chose a delay time of 13 to $17 \mathrm{~min}$ after contrast medium application, as reported in the literature.

\section{Contrast-enhanced MR angiography}

Parallel imaging techniques with an acceleration factor of 2 were applied for acquisition of all vascular territories. This resulted in an isotropic spatial resolution below $1.5 \mathrm{~mm}^{3}$ for the images of each station. We decided not to include coronary artery angiography in our study protocol, as this promising technique has not yet been introduced in the routine clinical setting $[9,10]$. MR angiography was performed using a standard 0.5-molar Gd contrast agent (gadopentetatedimeglumine, Magnevist, Schering, Berlin, Germany). The wb-MRA examination was divided into five stations in group 1 and four stations in group 2, respectively. Classification of arterial stenoses was as follows: mild stenosis $\leq 50 \%$, moderate stenosis $50-70 \%$, and high-grade stenosis $\geq 70 \%$ luminal narrowing. The first station included the aortic arch and the supra-aortic arteries. The second station imaged the abdominal aorta including the renal and iliac arteries. MRA of the lower extremities was divided into three and two steps for group 1 and 2, respectively. In subjects examined with the standard MR scanner (group 1), two different contrast injections were used to first image station 1; after repositioning of the individual, a second injection was used for stations 2 to 5. A biphasic injection protocol was used for the second injection with the first bolus consisting of $10 \mathrm{ml}$ of contrast agent at a rate of $1 \mathrm{ml} / \mathrm{s}$, followed by a bolus of $15 \mathrm{ml}$ at a flow rate of $0.5 \mathrm{ml} / \mathrm{s}$. For whole-body MRA of subjects in group 2 imaged with a dedicated whole-body MR system, two injections of contrast agent were also needed [26]. However, in this protocol, injection 1 was used to acquire stations 1 and 4 (carotid and calf arteries), while the second injection of contrast material was used for imaging of stations 2 and 3. This dedicated injection and acquisition protocol was used to minimise venous contamination while keeping the total amount of contrast agent as low as possible [24, 25].
Two radiologists with more than 6 years of experience in cardiovascular MRI evaluated the data individually. Findings were discussed and recorded according to consensus practice.

\section{Results}

All subjects successfully completed the wb-MRA scan. No adverse events occurred. In $31 \%$ of all participants, imaging revealed findings that led to further exams or a therapeutic intervention, e.g., starting a secondary preventive medication.

\section{Wb-MRA vascular system}

Of all study participants, $26.8 \%$ showed pathological changes in at least one of the examined vessel segments (Fig. 1). In 18 individuals $(7.2 \%)$, relevant atherosclerosis was found, i.e., at least a moderate stenosis (Fig. 2). In 39 subjects, atherosclerotic changes were present in one vascular territory. In a further 25 subjects (18.1\%), stenosis was found in more than one arterial segment (Fig. 3). A maximum of five arterial vascular regions were affected ( 2 cases, $1.4 \%)$. Regarding carotid arteries, stenoses were observed in seven cases $(4.9 \%)$. Of these stenoses, four were mild and two were moderate; in one case a higher grade stenosis of the proximal internal carotid artery was diagnosed. In the aortic region, in six cases plaques were observed, while the iliac arteries presented plaques in four cases $(2.9 \%)$, but without relevant luminal narrowing. In total, there were ten mild-grade stenoses, seven medium-grade stenoses, and seven high-grade stenoses within the large arterial vessels (Fig. 4). Furthermore, we found two low-grade aortic aneurysms. Additionally, we diagnosed one infrarenal aortic dissection and a dissection of the superficial femoral artery. Considering renal arteries, one subject showed mild-grade stenosis, while another one had plaques without luminal narrowing.

The most frequent significant atherosclerotic abnormalities ( $>50 \%$ stenosis) were found in the lower leg, namely 12

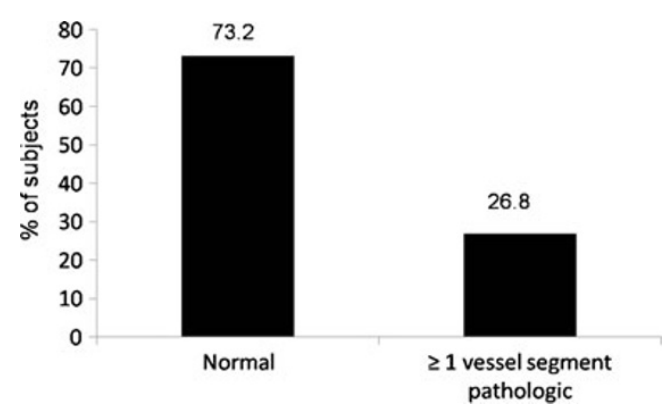

Fig. 1 Percentage of subjects with at least one pathologically modified vessel segment 


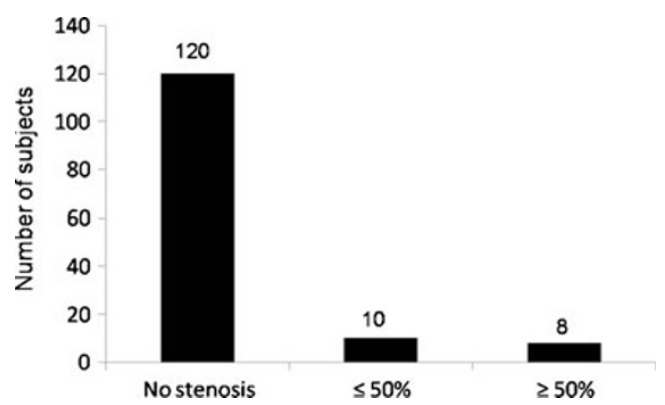

Fig. 2 Distribution of significant luminal narrowing in the present study population: $7.2 \%$ of the individuals showed mild to moderate luminal narrowing in at least one vascular territory; another $5.8 \%$ had at least one stenosis of $\geq 50 \% ; 86.9 \%$ presented without any stenoses

individuals presenting with mild $(2,1.4 \%$ of all subjects respectively), moderate $(3,2.2 \%)$, or severe stenosis $(7,5.0 \%)$. The detailed occurrences of low-, medium-, and high-grade stenoses referring to the four vascular territories (carotis, renal, femoral, and lower limb arteries) are presented in Figs. 1, 2, 3, 4 , and 5. The distribution of all pathological findings in the different vascular territories is summarised in Table 5 .

\section{Cardiac MR imaging}

Myocardial hypertrophy was present in four cases. Wall motion abnormalities were visually detected in six individuals $(4.3 \%)$, while in one case, the examination was judged not to be diagnostic. Subendocardial delayed contrast enhancement was found in two cases $(1.4 \%)$. A perfusion deficit was present in 13 cases $(9.1 \%)$; for an example, see Fig. 6. Perfusion deficits related to myocardial hypertrophy were found in four cases. In five males, a regional $(n=4)$ or global $(\mathrm{n}=1)$ myocardial dysfunction with an ejection fraction $<50 \%$ was present. Nine subjects showed valve diseases $(6.4 \%)$ as visually detected in the functional evaluation of the

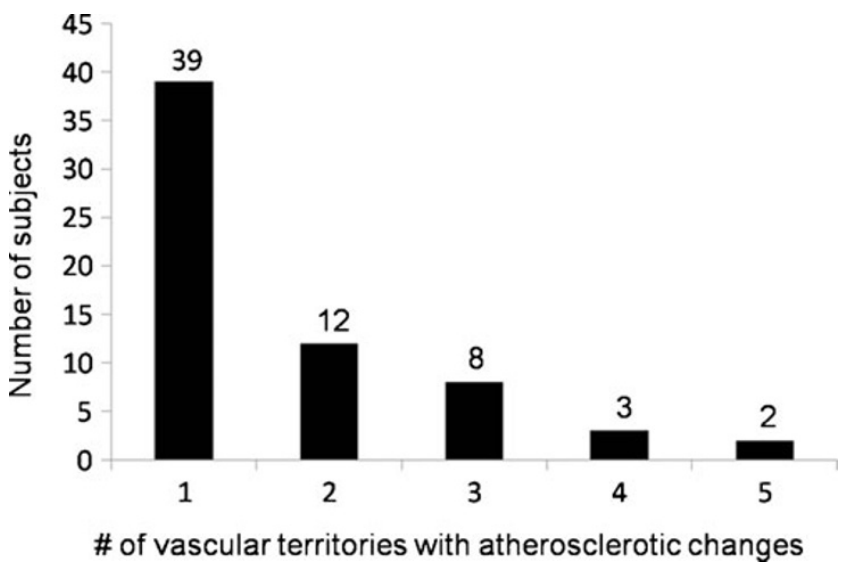

Fig. 3 Number of vascular territories (carotid arteries, iliacal arteries, aorta, femoral arteries, tibial and fibular arteries) that presented with atherosclerotic changes (plaques, luminal narrowing, dissection, aneurysm) in one subject at a time

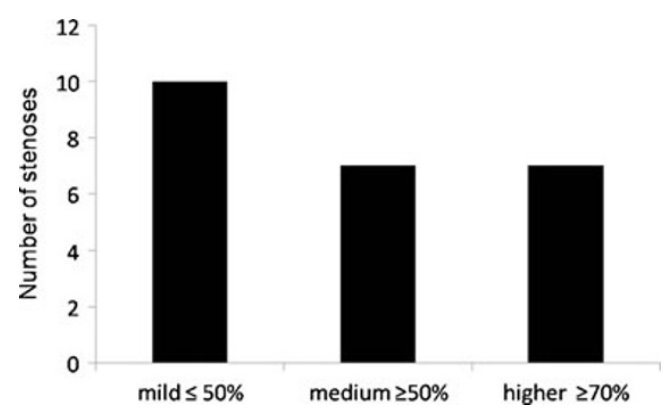

Fig. 4 Number of stenoses of mild, medium, and higher grade found in all participants

left ventricular myocardium, with two subjects displaying findings consistent with aortic valve insufficiency, two with aortic stenosis, and two with mitral valve insufficiency. In one case, signs of pericarditis were found.

\section{Additional findings}

Apart from the main target diseases, there were several incidental findings, as listed in Table 6. In the pulmonary examination, five cases of pathologically enlarged lymphatic nodules were detected. In addition, there were four inflammatory nodules, one inflammatory infiltrate, and one nodule of primarily unspecifiable origin. Concerning artheriosclerotic lesions, in three cases, cerebral microangiopathy was present. In nine further examinations $(6.5 \%)$, we observed unspecific cerebral white-matter lesions, most likely due to microangiopathy. In another single case, a meningioma (Fig. 7) was detected. No malignant lesions were discovered using this cardiovascular whole-body MRI protocol.

\section{Discussion}

This study showed that wb-MRI can be performed and evaluated with the use of 1.5-T clinical MR systems. The feasibility of whole-body cardiovascular MRI has been

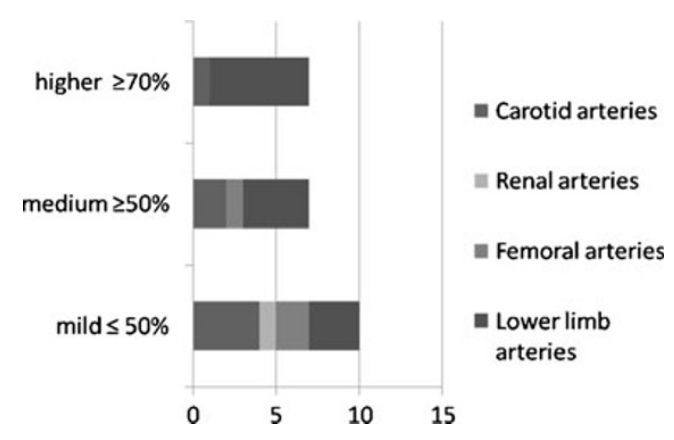

Fig. 5 Degree of the luminal narrowing encountered. In the iliac arteries, no relevant luminal narrowing was found 
Table 5 Distribution of pathological findings and nonevaluable sections in the vascular territories examined

CAR, carotid arteries; AOR, aorta; REN, renal

arteries; ILI, iliacal arteries;

FEM femoral arteries; POP, popliteal artery, PTA, posterior tibial artery; TPT,

tibioperoneal trunk

\begin{tabular}{|c|c|c|c|c|c|c|}
\hline & CAR & AOR & REN & ILI & FEM & POP/PTA/TPT \\
\hline Low grade & 4 & & 1 & & 2 & 3 \\
\hline Medium-grade stenosis & 2 & & & & 1 & 4 \\
\hline Higher grade stenosis & 2 & & 1 & & & 6 \\
\hline Plaques & 3 & & 1 & 4 & & 1 \\
\hline Elongation & 5 & 4 & 1 & 2 & 2 & \\
\hline Arterio-venous shunt & 1 & & & & & \\
\hline Aneurysm & & 2 & & & & \\
\hline Unevaluable & & & & 1 & & 1 \\
\hline \multicolumn{7}{|l|}{ Motion } \\
\hline \multicolumn{7}{|l|}{ Venous overlay } \\
\hline Lack of contrast & & & & & & \\
\hline
\end{tabular}

shown by several authors. General sensitivity and specificity have been determined to be between 91 to $95.3 \%$ and 90 to $95.2 \%$, respectively $[4,5]$. Until now, only a limited number of studies with larger study cohorts consisting of healthy subjects have been published [11-13]. Study collectives differ considerably; thus, caution has to be exercised when comparing results between different studies. Our study provides useful information about the prevalence of cardiovascular disease in a large, relatively homogenous collective. Therefore, it contributes data to assess the significance of wb-MRI/-MRA in broad clinical use as a diagnostic screening tool. We observed vascular pathology in more than $26 \%$ of asymptomatic subjects, and this may support the secondary preventive use of wb-MRI/-MRA in a preselected population.

Nevertheless, until now, we do not have sufficient longterm outcome data to evaluate whole-body MRI as a screening tool compared to other measures of secondary prevention. In the future, prospective studies need to be performed

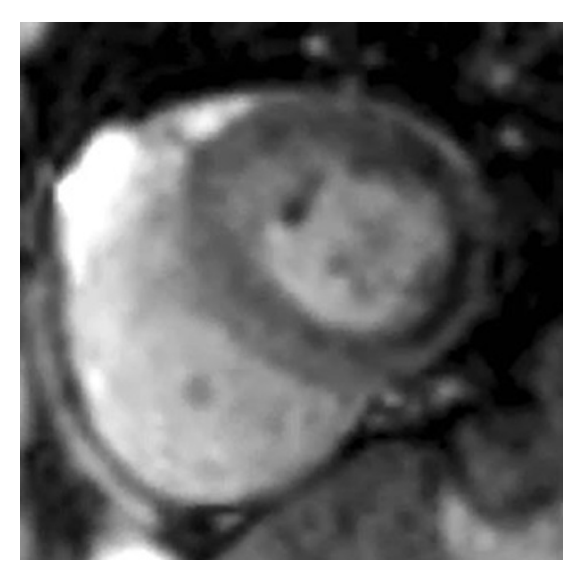

Fig. 6 Myocardium showing a cardiac perfusion deficit detected by use of the saturation-recovery trueFISP sequence; parallel imaging factor of 2 to answer this question. At the same time, the costeffectiveness of the method must be investigated.

Hansen et al. reported on wb-MRA for screening for atherosclerotic disease in 307 elderly patients. In Hansen's collective, two-thirds of the subjects had vascular abnormalities, of which one third showed stenosis of less than $50 \%$ and a third was found to have luminal narrowing greater than 50\%. The remaining individuals showed no vascular abnormalities. Whereas in our cohort only $37 \%$ had vascular pathologies, one has to consider that the median age of Hansen's cohort was 14 years older than the median age of our participants, in addition to having a different distribution of males and females. According to our results, most of relevant luminal narrowing detected by Hansen was located in the arteries of the lower legs.

Whenever we are investigating subjects with known underlying factors predisposing to atherosclerotic changes, a much higher prevalence of manifest arteriosclerosis is found. Weckbach et al. found evidence for cardiovascular disease in patients with long-term histories of diabetic disease in $49 \%$ of cases for peripheral artery disease, in $25 \%$ of cases for myocardial infarction, and in $28 \%$ of cases for cerebrovascular disease [14]. Ladd et al. analyzed patients with coronary artery disease (CAD) for additional signs of arteriosclerosis. Ten percent of the total group had symptoms of stroke once in their lifetime; $48.1 \%$ had luminal narrowing greater than $50 \%$ in at least one extracardial artery [15]. In 60 study subjects with known peripheral arterial occlusive disease, Fenchel et al. [16] found 38 subjects with myocardial dys-, hypo-, or akinesia (63.3\%). This is a considerably higher percentage than the $4.3 \%$ we observed in our collective. Cardiac delayed contrast enhancement was present in $24(40 \%)$ cases versus $2(1.4 \%)$ cases in our study and cerebral ischemia in $18(30 \%)$ cases vs. 1 $(0.7 \%)$ case. Significant cerebral microangiopathy was present in seven (11.7\%) cases vs. none in our collective. 
Table 6 Number and characteristics of findings apart from the main target diseases

\begin{tabular}{|c|c|c|c|c|c|}
\hline \multirow[t]{2}{*}{ Technique } & \multicolumn{3}{|l|}{ Incidental findings on MRI } & \multicolumn{2}{|l|}{ Of those } \\
\hline & & $\mathrm{n}$ & $\%$ & $\begin{array}{l}\text { Relevant } \\
\text { findings (n) }\end{array}$ & $\begin{array}{l}\text { Non-relevant } \\
\text { findings (n) }\end{array}$ \\
\hline \multirow[t]{8}{*}{ Cerebral MRI } & Gliosis & 6 & 4.3 & & 6 \\
\hline & White-matter lesions & 9 & 6.5 & 9 & \\
\hline & Meningioma & 1 & 0.7 & 1 & \\
\hline & Unspecified lesion & 10 & 7.2 & & 10 \\
\hline & Microangiopathy & 3 & 2.2 & 3 & 0 \\
\hline & Atypical vessels & 1 & 0.7 & & 1 \\
\hline & Virchow-Robin spaces dilated & 7 & 4.7 & & 7 \\
\hline & Arachnoid cyst & 2 & 1.4 & & 2 \\
\hline \multirow[t]{2}{*}{ MR angiography } & Ulcer of aortic wall & 1 & 0.7 & 1 & \\
\hline & Compression of celiactrunc & 2 & 1.4 & 2 & \\
\hline \multirow[t]{13}{*}{ Pulmonary MRI } & Mediastinal lymph nodes enlarged & 1 & 0.7 & 1 & \\
\hline & Hilar lymph nodes enlarged & 1 & 0.7 & 1 & \\
\hline & Paratracheal lymph nodes enlarged & 1 & 0.7 & 1 & \\
\hline & Axillary lymph nodes enlarged & 2 & 1.4 & 2 & \\
\hline & Total lymph nodes enlarged & 5 & 3.5 & 5 & \\
\hline & Pleural scars & 1 & 0.7 & 1 & \\
\hline & Encapsulated effusion & 1 & 0.7 & 1 & \\
\hline & Pleural layers & 1 & 0.7 & 1 & \\
\hline & Cyst & 1 & 0.7 & 1 & \\
\hline & Azygos lobe & 1 & 0.7 & & 1 \\
\hline & Inflammatory nodule & 4 & 2.9 & 1 & \\
\hline & Nodule of unknown origin & 1 & 0.7 & 1 & \\
\hline & Inflammatory infiltration & 1 & 0.7 & 1 & \\
\hline \multirow[t]{7}{*}{ Cardiac MRI } & Aortic stenosis, low grade & 2 & 1.4 & 2 & \\
\hline & Aortic insufficiency & 2 & 1.4 & 2 & \\
\hline & Mitral insufficiency & 1 & 0.7 & 1 & \\
\hline & Other valve abnormalities & 4 & 2.9 & 4 & \\
\hline & Hypertrophy of the left ventricle & 2 & 1.4 & 2 & \\
\hline & Pericarditis & 1 & 0.7 & 1 & \\
\hline & Pericardic cyst & 1 & 0.7 & 1 & \\
\hline \multirow[t]{3}{*}{ MRI abdomen } & Hepatic cysts & 3 & 2.1 & 3 & \\
\hline & Hepatic hemangioma & 4 & 2.8 & 4 & \\
\hline & $\begin{array}{l}\text { Chronic hepatic parenchymal defect } \\
\text { (cirrhosis, fatty liver, ascites) }\end{array}$ & 2 & 1.4 & 2 & \\
\hline
\end{tabular}

The prevalence rates for the presence of atherosclerosis and its complications observed in our study population without a prior history of vascular disease are comparable to data known from large epidemiological studies on middle-aged subjects. Goehde et al. found relevant pathological findings in 90 out of 175 asymptomatic individuals (51.4\%) who underwent wb-MRI/-MRA. In previous studies performed with ultrasound in asymptomatic populations, the prevalence of atherosclerosis in the extracranial carotid artery ranged from 5.9 to $32.8 \%$. Carotid artery stenoses of at least $50 \%$ were found in 4 to $7.7 \%[17,18]$.
Functional cardiac analysis in the present study found wall motion abnormalities in $3.6 \%$ of all study participants and cardiac perfusion deficits in 12 cases $(8.7 \%)$, as illustrated by an example in Fig. 6. In this subgroup, the results for evidence of clinically unrecognized myocardial infarctions in healthy individuals seem relatively high compared to the results of Goehde et al., who detected visual wall motion abnormalities in 5 of 298 (1.7\%) screening participants. This might be due to the combination of three different examinations in our study, including DCE, functional wall motion analysis, 


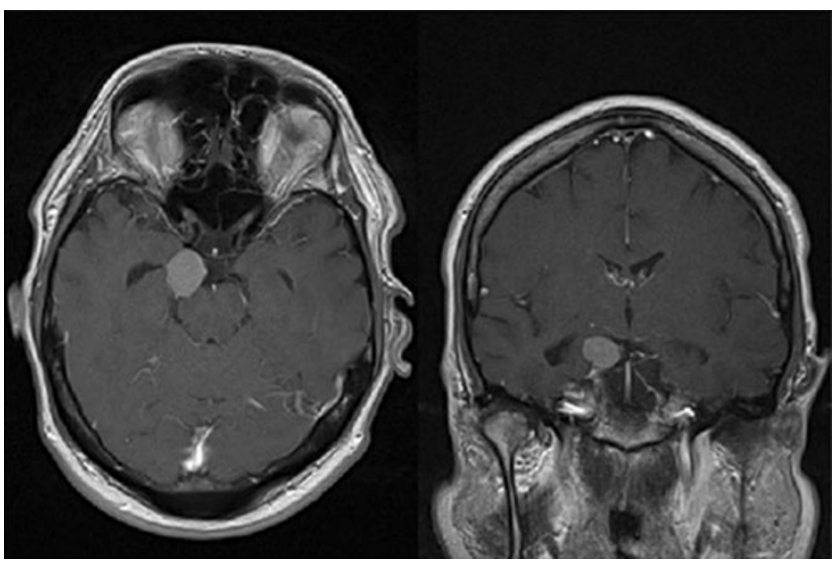

Fig. 7 Incidental meningioma parapontine

and perfusion analysis. In agreement with this, Breuckmann and colleagues described clinically asymptomatic delayed cardiac enhancement in $4 \%$ of a group of 102 individuals [19].

Another whole-body MRI study by Hegenscheid et al. included 200 healthy volunteers. With a median age of 48.3 years, the mean age of this cohort was lower than in our collective. In 176 subjects (88\%), they detected 431 pathological findings, of which $45(10.4 \%)$ required further clinical workup. In comparison, findings that necessitated further diagnostic workup or therapeutic steps were found in $31 \%$ of our volunteers.

In order to keep the scan time within an acceptable range (below $90 \mathrm{~min}$ ), we did not evaluate the colon and intestine in our protocol, although this has been done by other groups such as Goehde et al. to screen for colon cancer and polyps [11]. With regard to the tumor screening part of their wbMRI study, they found colon polyps in 12 cases (4.1\%) and one incidental renal cell carcinoma. The majority of reports in the literature on wb-MRI as a screening tool for malignancy describe it as a screening tool for metastasis detection in known cancer patients, e.g., with breast or prostate cancer. MRI has been found to be equivalent regarding the detection of bone metastases compared to scintigraphy and FDG-PET [20, 21].

Whole-body MRI/MRA is a safe, reproducible technique with few side effects. In a collective of middle-aged individuals, more than one third of the participants showed previously unknown, in part therapeutically relevant findings. These findings led to an application of additional diagnostic measures or intensified control intervals in our study subjects. Therefore, in combination with the modification of cardiovascular risk factors, possibly fatal cardiovascular events might be prevented $[1,2]$.

Incidental findings were categorised as important/necessitating treatment or as incidental findings without the need for immediate/further treatment. If important findings were detected, those were immediately reported to the referring physician. Incidental findings without the need for further action were described in the written report.

There are various limitations to our study: first, we are subject to a certain selection bias as our study cohort consisted of employees from two big companies with the majority being male participants. However, female participants were not excluded from the evaluation since they also provide data on the presence of atherosclerotic changes in women compared to men. Study participants were relatively young with a mean age of 56 years compared to study subjects in comparable studies [11, 13, 14, 28]. The cumulative prevalence of peripheral arterial disease is estimated to be between 3 to $10 \%$ in epidemiological studies. By the age of 70 or more, the prevalence increases up to $15-20 \%$ $[22,23]$. Second, a major limitation of this study is the lack of a gold standard to objectively evaluate the diagnostic accuracy of this wb-MRI/-MRA protocol. On the other hand, a preliminary study performed in our department with the same MR protocol showed not only good routine applicability, but could also confirm pathological findings on MRI/MRA by further diagnostic measures on a case-tocase basis [12]. However, the gold standard for the examination of atherosclerotic disease is still digital substraction angiography (DSA) or, in certain arterial territories, duplex ultrasound. In a collective of apparently healthy individuals, we had to avoid the use of ionizing radiation associated with DSA or CT as comparative methods. Ultrasound of arterial vessels would be a suitable alternative to conventional angiography. It is, however, limited to the visualisation of several well-accessible vascular regions such as the carotid arteries and the arteries of the upper legs. It is still time consuming and dependent on the examiner's experience. Further, MR angiography is not the method of choice to describe vessel wall changes such as distinguishing soft and hard plaques. In well-accessible territories, duplex ultrasound may be more useful to detect such changes. Importantly, although whole-body MRA is accurate for the detection of atherosclerotic disease, no prospective studies have shown the benefit of interventions based on wholebody MRA findings. In order for wb-MRA to become widely accepted as a screening tool, outcome studies are needed.

In summary, wb-MRI and wb-MRA comprise a reliable, reproducible and easily applicable diagnostic tool for the detection of atherosclerotic disease in all major arterial territories. However, there is little probability for malignant disease in a cohort of asymptomatic subjects. Whenever there were suspicious findings in primary wbMRI, an additional reference examination, such as a dedicated abdominal MRI, for further lesion characterisation was recommended. 
Open Access This article is distributed under the terms of the Creative Commons Attribution License which permits any use, distribution, and reproduction in any medium, provided the original author(s) and the source are credited.

\section{References}

1. Ford ES, Ajani UA, Croft JB, Critchley JA, Labarthe DR, Kottke TE, Giles WH, Capewell S (2007) Explaining the decrease in US deaths from coronary disease, 1980-2000. N Engl J Med 7 (356):2388-2398

2. Palmieri L, Bennett K, Giampaoli S, Capewell S (2010) Explaining the decrease in coronary heart disease mortality in Italy between 1980 and 2000. Am J Public Health 100:684-692

3. Diehm N, Diehm C (2006) Subclinical atherosclerosis of lower limb arteries: a strong predictor for cardiovascular mortality. Eur Heart J27:2495-2496

4. Ruehm SG, Goyen M, Barkhausen J, Kröger K, Bosk S, Ladd ME, Debatin JF (2001) Rapid magnetic resonance angiography for detection of atherosclerosis. Lancet 7(357):1086-1091

5. Goyen M, Quick H, Debatin JF, Ladd ME, Barkhausen J, Herborn CU, Bosk S, Kuehl H, Schlepütz M, Ruehm SG (2002) Whole body Three-dimensional MR angiography with a rolling table platform: initial clinical experience. Radiology 224:270-277

6. Miller S, Simonetti OP, Carr J, Kramer U, Finn JP (2002) MR Imaging of the heart with cine true fast imaging with steady-state precession: influence of spatial and temporal resolutions on left ventricular functional parameters. Radiology 223:263-269

7. Kellman P, Arai A, McVeigh E (2002) Phase-sensitive inversion recovery for detecting myocardial infarction using gadoliniumdelayed hyperenhancement. Magn Reson Med 47:372-383

8. Huber AM, Schoenberg SO, Hayes C, Spannagl B, Engelmann MG, Franz WM, Reiser MF (2005) Phase-sensitive inversionrecovery MR imaging in the detection of myocardial infarction. Radiology 237:854-860

9. Ladd S, Ladd M (2007) Perspectives for preventive screening with total body MRI. Eur Radiol 17:2889-2897

10. Prompona M, Cyran C, Nikolaou K, Bauner K, Reiser M, Huber A (2009) Contrast-enhanced whole-heart MR coronary angiography at $3.0 \mathrm{~T}$ using the intravascular contrast agent gadofosveset. Invest Radiol 44:369-374

11. Goehde S, Hunold P, Vogt FM, Ajaj W, Goyen M, Herborn CU, Forsting M, Debatin JF, Ruehm SG (2005) Full-body cardiovascular and tumor MRI for early detection of disease: feasibility and initial experience in 298 subjects. AJR 184:598-611

12. Kramer H, Schoenberg SO, Nikolaou K, Huber A, Struwe A, Winnik E, Wintersperger BJ, Dietrich O, Kiefer B, Reiser MF (2005) Cardiovascular screening with parallel imaging techniques and a whole-body MR imager. Radiology 236:300-310

13. Hansen T, Wikström J, Johansson LO, Lind L, Ahlström H (2007) The prevalence and quantification of atherosclerosis in an elderly population assessed by whole-body magnetic resonance angiography. Arterioscler Thromb Vasc Biol 27:649-654

14. Weckbach S, Findeisen H, Schoenberg SO, Kramer H, Stark R, Clevert DA, Reiser MF, Parhofer KG (2009) Systemic cardiovas- cular complications in patients with long-standing diabetes mellitus: comprehensive assessment with whole-body magnetic resonance imaging/magnetic resonance angiography. Invest Radiol 44:242-250

15. Ladd S, Debatin J, Stang A et al (2007) Whole-body MR vascular screening detects unsuspected concomitant vascular disease in coronary heart disease patients. Eur Radiol 17:1035-1045

16. Fenchel M, Jost D, Kramer U, Stauder N, Bretschneider C, Klumpp B, Scheule A, Nägele T, Claussen CD, Miller S (2006) Cardiovascular whole-body MR imaging in patients with symptomatic peripheral arterial occlusive disease. Rofo 178:491-499

17. Pujia A, Rubba P, Spencer MP (1992) Prevalence of extracranial carotid artery disease detectable by echo-Doppler in an elderly population. Stroke 23:818-822

18. Colgan MP, Strode GR, Sommer JD, Gibbs JL, Sumner DS (1988) Prevalence of asymptomatic carotid disease: results of duplex scanning in 348 unselected volunteers. J Vasc Surg 8:674-678

19. Breuckmann F, Möhlenkamp S, Nassenstein K, Lehmann N, Ladd S, Schmermund A, Sievers B, Schlosser T, Jöckel KH, Heusch G, Erbel R, Barkhausen J (2009) Myocardial late gadolinium enhancement: prevalence, pattern, and prognostic relevance in marathon runners. Radiology 251:50-57

20. Schmidt GP, Schoenberg SO, Schmid R, Stahl R, Tiling R, Becker CR, Reiser MF, Baur-Melnyk A (2007) Screening for bone metastases: whole-body MRI using a 32-channel system versus dualmodality PET-CT. Eur Radiol 17:939-949

21. Lauenstein T, Freudenberg LS, Goehde SC, Ruehm SG, Goyen M, Bosk S, Debatin JF, Barkhausen J (2002) Whole-body MRI using a rolling table platform for the detection of bone metastases. Eur Radiol 12:2091-2099

22. Criqui MH, Fronek A, Barrett-Connor E, Klauber MR, Gabriel S, Goodman D (1985) The prevalence of peripheral arterial disease in a defined population. Circulation 71:510-515

23. Diehm C, Schuster A, Allenberg J, Darius H, Haberl R, Lange S, Pittrow D, von Stritzky B, Tepohl G, Trampisch HJ (2004) High prevalence of peripheral arterial disease and comorbidity in 6880 primary care patients: cross sectional study. Atherosclerosis 172:95-105

24. Huber A, Schoenberg SO, Spannagl B, Rieber J, Erhard I, Klauss V, Reiser MF (2006) Single-shot inversion recovery TrueFISP for assessment of myocardial infarction. AJR Am J Roentgenol 186:627-633

25. Kramer H, Nikolaou K, Reiser MF (2009) Cardiovascular wholebody MRI. Eur J Radiol 70:418-423

26. Klessen C, Asbach P, Hein PA, Lembcke A, Bauknecht HC, Huppertz A, Nentwig T, Hamm B, Taupitz M (2006) Wholebody MR angiography: comparison of two protocols for contrast media injection. Rofo 178:484-490

27. Roger L, Go A, Lloyd-Jones D (2012) Executive summary: Heart Disease and Stroke Statistics 2012 Update: a report from the American Heart Association. Circulation 125:188-197

28. Hegenscheid K, Kühn JP, Völzke H, Biffar R, Hosten N, Puls R (2009) Whole-body magnetic resonance imaging of healthy volunteers: pilot study results from the population-based SHIP study. Rofo 181(8):748-759 